\title{
CFD Simulation of Proposed Validation Data for a Flow Problem Reconfigured to Eliminate an Undesirable Flow Instability
}

\section{FEDSM-ICNMM 2010}

Richard W. Johnson

Hugh M. Mcllroy

The INL is a

U.S. Department of Energy

National Laboratory

operated by

Battelle Energy Alliance

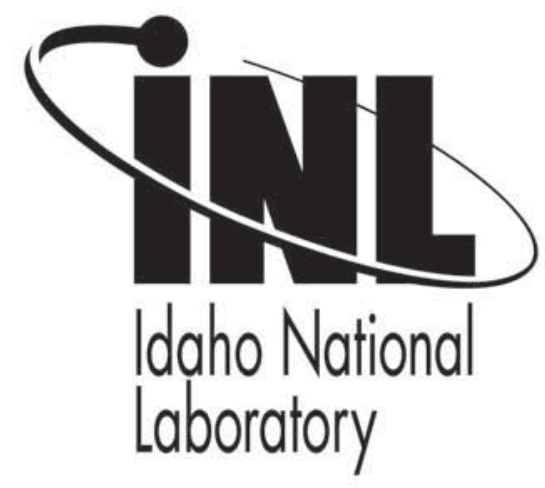

This is a preprint of a paper intended for publication in a journal or proceedings. Since changes may be made before publication, this preprint should not be cited or reproduced without permission of the author. This document was prepared as an account of work sponsored by an agency of the United States Government. Neither the United States Government nor any agency thereof, or any of their employees, makes any warranty, expressed or implied, or assumes any legal liability or responsibility for any third party's use, or the results of such use, of any information, apparatus, product or process disclosed in this report, or represents that its use by such third party would not infringe privately owned rights. The views expressed in this paper are not necessarily those of the United States Government or the sponsoring agency. 


\section{FEDSM-ICNMM2010-30522}

\section{CFD SIMULATION OF PROPOSED VALIDATION DATA FOR A FLOW PROBLEM RECONFIGURED TO ELIMINATE AN UNDESIRABLE FLOW INSTABILITY}

\author{
Richard W. Johnson \\ Idaho National Laboratory \\ Idaho Falls, Idaho, USA \\ Rich.Johnson@inl.gov
}

\author{
Hugh M. Mcllroy \\ Idaho National Laboratory \\ Idaho Falls, Idaho, USA \\ Hugh.Mcllroy@inl.gov
}

\section{ABSTRACT}

The U. S. Department of Energy (DOE) is supporting the development of a next generation nuclear plant (NGNP), which will be based on a very high temperature reactor (VHTR) design. The VHTR is a single-phase helium-cooled reactor wherein the helium will be heated initially to $750{ }^{\circ} \mathrm{C}$ and later to temperatures approaching $1000{ }^{\circ} \mathrm{C}$. The high temperatures are desired to increase reactor efficiency and to provide a heat source for the manufacture of hydrogen and other applications. While computational fluid dynamics (CFD) has not been used in the past to design or license nuclear reactors in the U. S., it is expected that CFD will be used in the design and safety analysis of forthcoming designs. This is partly because of the maturity of CFD and partly because detailed information is desired of the flow and heat transfer inside the reactor to avoid hot spots and other conditions that might compromise reactor safety. Numerical computations of turbulent flow should be validated against experimental data for flow conditions that contain some or all of the physics expected in the thermal fluid machinery of interest. To this end, a scaled model of a narrow slice of the lower plenum of the prismatic VHTR was constructed and installed in the Idaho National Laboratory's (INL) matched index of refraction (MIR) test facility and data were taken. The data were then studied and compared to CFD calculations to help determine their suitability for validation data. One of the main findings was that the inlet data, which were measured and controlled by calibrated mass flow rotameters and were also measured using detailed stereo particle image velocimetry (PIV) showed considerable discrepancies in mass flow rate between the two methods. The other finding was that a randomly unstable recirculation zone occurs in the flow. This instability has a very significant effect on the flow field in the vicinity of the inlet jets. Because its time scale is long and because it is apparently a random instability, it was deemed undesirable for a validation data set. It was predicted using
CFD that by eliminating the first of the four jets, the recirculation zone could be stabilized. The present paper reports detailed results for the three-jet case with comparisons to the four-jet data inasmuch as three-jet data are still unavailable. Hence, the present simulations are true or blind predictions.

\section{INTRODUCTION}

The core of the prismatic VHTR is characterized by hexagonal graphite blocks machined to allow insertion of nuclear fuel compacts surrounded by coolant channels to convect away the heat. The coolant channels empty into the lower plenum as downward flowing jets that then turn $90^{\circ}$ toward the exit duct. The helium then flows past many graphite support columns. A 1:6.55 scaled model of a slice of the lower plenum was constructed from quartz. The model contains several posts and is fed by vertical inlet ducts representing helium jets. Figure 1 is a drawing of the scaled model.

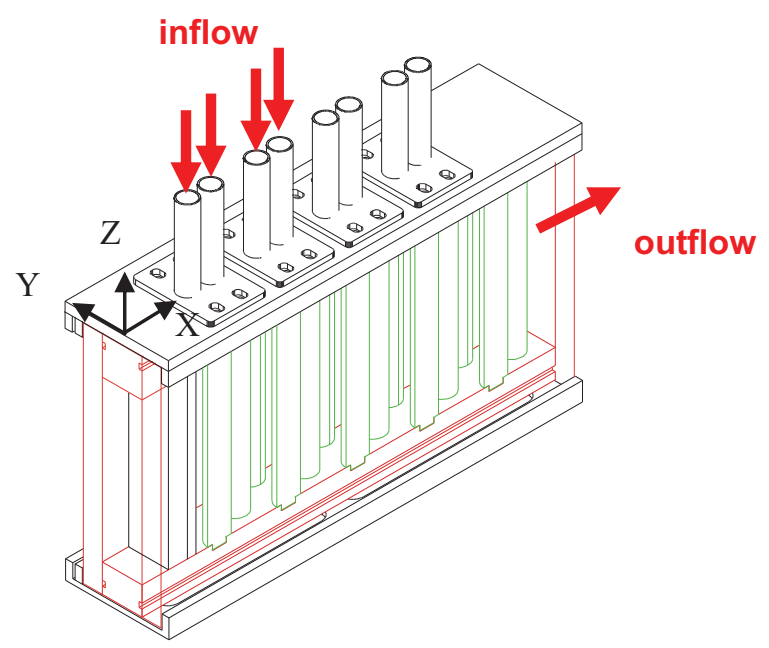

Figure 1. Isometric view of the scaled model. 
Flow data were taken in the scaled model using stereo particle image velocimetry (PIV) [1] in the INL's matched index of refraction (MIR) facility. The flow into each of the four inlet ducts was adjusted and monitored using calibrated mass flow rotameters, which, of course, simply measured the mass flow rate. Regions of the inlet ducts above the top plane of the lower plenum were visible such that PIV measurements of the inlet flow could be also taken. Detailed measurements of the inlet flow are desirable because all three velocity components plus turbulent kinetic energy can be measured.

Inlet data were extracted from the PIV data at $Z=9.7$ and $11 \mathrm{~mm}$, including the three mean velocity components and the turbulent kinetic energy (TKE). The PIV data were read into the commercial CFD code STARCCM+ [2] and then interpolated onto the mesh by the code. The data were integrated by the CFD code to obtain the mass flow rates for each inlet duct. It was found that there were significant discrepancies between the mass flows based on the PIV data at the two locations and the mass flows measured directly by the rotameters [3]. Table 1 gives the percentage differences between the PIV-based mass flows and the rotameter data. The rotameter data are deemed the most accurate as the rotameters were each calibrated. The PIVbased inlet mass flows are all too low as seen in Table 1. Also, there are significant discrepancies between the two PIV-based datasets.

Table 1. Percent variation of PIV from rotameter data.

\begin{tabular}{|c|c|c|c|c|c|}
\hline $\begin{array}{c}\text { PIV } \\
\text { data }\end{array}$ & Jet 1 & Jet 2 & Jet 3 & Jet 4 & Overall \\
\hline $9.7 \mathrm{~mm}$ & $\begin{array}{c}25 . \% \\
\text { low }\end{array}$ & $\begin{array}{c}8.0 \% \\
\text { low }\end{array}$ & $\begin{array}{c}4.5 \% \\
\text { low }\end{array}$ & $\begin{array}{c}9.0 \% \\
\text { low }\end{array}$ & $\begin{array}{c}10.4 \% \\
\text { low }\end{array}$ \\
\hline $11 \mathrm{~mm}$ & $\begin{array}{c}18 . \% \\
\text { low }\end{array}$ & $\begin{array}{c}0.8 \% \\
\text { low }\end{array}$ & $\begin{array}{c}3.0 \% \\
\text { low }\end{array}$ & $\begin{array}{c}8.8 \% \\
\text { low }\end{array}$ & $\begin{array}{c}6.7 \% \\
\text { low }\end{array}$ \\
\hline
\end{tabular}

In view of the discrepancies between the rotameter and the PIV data for the inlet mass flows, it was decided that the best approach to setting the inlet flow conditions for the CFD simulation was to use the rotameter data and set the inlet velocity to the uniform value computed for the bulk velocity. The inlet plane was set to the maximum height of the inlet ducts, $88.5 \mathrm{~mm}$; the two tangential components were set to zero. The inlet TKE was set to a value such that the value measured by PIV was matched in the core of the inlet flow at $Z=9.7 \mathrm{~mm}$. Improved results were obtained for these inlet conditions, as reported in Ref. [4]. However, it was also discovered that an undesirable flow condition was present in the flow of the scaled model [4]. This was a recirculation zone below Jet 1 that was unstable with a characteristic time an order of magnitude greater than the vortex shedding behind the posts inside the model. This unstable zone has a very significant impact on the flow field inside the model; it was determined computationally that turning off Jet 1 should render the recirculation zone stable
[4]. It is expected to be able to obtain new MIR data for the scaled model of the VHTR lower plenum with improved PIVbased inlet data and with the first jet turned off. In anticipation of the new data, computations have been made for four turbulence models with Jet 1 turned off and using the best inlet conditions from the above four-jet MIR data for the three remaining jets. These computations represent true predictions because the data have not yet been taken; the computations can also be termed blind predictions.

\section{CFD MODEL}

The scaled model of a slice of the VHTR lower plenum has several inlet jets and a host of full and half cylinders that represent the graphite support columns that hold up the core as can be seen in Figure 2. However, for the present calculations, the first jet at the left, which is centered on the point of the wedge has been shut off.

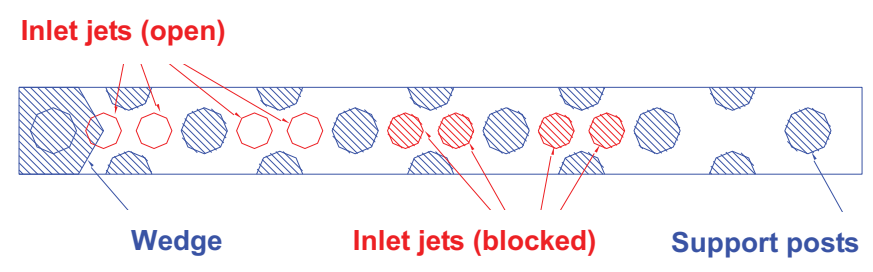

Figure 2. Plan view of the scaled model.

The scaled model has an overall length of $558.8 \mathrm{~mm}$, a height of $217.5 \mathrm{~mm}$ and a width of $54 \mathrm{~mm}$. The cylindrical jet inlets and support posts have diameters of 22.1 and $31.8 \mathrm{~mm}$, respectively. The posts are spaced $93.5 \mathrm{~mm}$ apart, while Jets 3 \& 4 are spaced $31.3 \mathrm{~mm}$ apart. A 3-D mesh was constructed based on earlier 2-D meshes that were used to look at the flow domain [5]. While the mesh was deemed somewhat coarse, it was found to be able to provide important results in the study of the MIR data [3-6]. The mesh, denoted mesh a4, is a hexahedral mesh, with 5.78 million cells, created using GAMBIT 2.4.6 [7]. Furthermore, it was shown in Ref. [5] that for a 2-D version of the problem, the CFD domain need only include the scaled model, and not the flow field around it if a pressure-outlet boundary condition is used at the outlet; the calculations were shown to be accurate just upstream of the outlet for the case of using only the domain of the model compared to that of including the actual outer flow field that was present in the experimental facility. This gave confidence that the same would apply for the 3-D case.

Turbulence models in STARCCM+ [2] employed in the present study include the default Reynolds stress transport model (RSM) with the two layer all $y+$ wall treatment, the standard $k \sim \varepsilon$ (SKE) two layer model with the all $y^{+}$wall model, the Abe-Kondoh-Nagano (AKN) $k \sim \varepsilon$ low Re with the all $y^{+}$wall model and the Menter shear-stress transport $k \sim \omega$ (SKW) model with the all $y^{+}$wall model. The all $y^{+}$wall treatment is a hybrid treatment that uses wall functions if the local mesh is too coarse 
to contain the viscous sublayer, but uses the appropriate low $\mathrm{y}^{+}$ treatment otherwise. The $y^{+}$values in the mesh at the several walls range from 1 to 12 . The mesh is mapped in the vicinity of the walls and paved elsewhere in the cross-sectional plane. The mesh was extruded in the vertical direction. The flow is considered to be a nonstationary flow inasmuch as there are fluctuations present that are not turbulent fluctuations; these are the vortex shedding behind the several full and half posts. Hence, the approach used is termed an unsteady Reynoldsaveraged Navier Stokes or URANS approach, where the describing Navier Stokes equations have been ensemble or Reynolds-averaged.

Mathematically, the instantaneous flow variables can be decomposed into an ensemble average and a random (turbulent) fluctuating component. That is, for the X-component velocity:

$u=<u>+u^{\prime}$

where $\langle u\rangle$ is the ensemble average and $u^{\prime}$ is the turbulent fluctuation. The ensemble average can vary in time. The ensemble-averaged quantity can be decomposed further into a time-averaged component and a coherent fluctuation. The coherent fluctuation is related to unsteadiness such as vortexshedding. That is

$<u>=U+\tilde{u}$

where $U$ is the time average of the ensemble average (or longtime average or just time mean) and $\tilde{u}$ is the coherent fluctuation. These averaging processes are applied to the velocity components and pressure. The velocity components are given as $u, v$ and $w$ in the $\mathrm{X}, \mathrm{Y}$ and $\mathrm{Z}$ directions, respectively, which are defined in Figure 1. The present CFD simulations compute the ensemble averages. These are concurrently timeaveraged because the MIR data are long-time averaged.

The commercial CFD code STAR-CCM+ [2] is used for the computations. Second-order differencing is used for both spatial and the implicit temporal discretizations; the segregated solver is used. Walls are considered smooth and are assigned the no-slip condition. Computations use a time step of $2.0 \times 10^{-4}$ second. The 'pressure-outlet' boundary condition is used at the outlet. The iterative convergence for each time step is set based on the calculation of laminar Poiseuille flow. It was found from the Poiseuille flow calculation that a residual computed in STARCCM + is converged for a residual of about $2 \times 10^{-4}$. Similar exercises showed that using Poiseuille flow to establish iterative convergence could be extended to an unsteady flow in a tube bank $[8,9]$. Initial conditions were set at stagnant values. The MIR data are for the case of jet inlet Reynolds number of 12,400 .

\section{RESULTS AND DISCUSSION}

Results are presented for the case of Jet 1 turned off (the three-jet case). The data used for comparison purposes are the MIR four-jet case. Figure 3 illustrates profile and point locations that are used for comparison purposes. The profiles will be referred to with two suffixes: ' $u$ ' and 'd.' Suffix ' $u$ ' (up) indicates that the plane of the profile is at $\mathrm{Z}=-70 \mathrm{~mm}$ (about $1 / 3$ the way from the top to the bottom plane); suffix ' $d$ ' (down) indicates that the profile is at $\mathrm{Z}=-150 \mathrm{~mm}$ (about $2 / 3$ the way down).

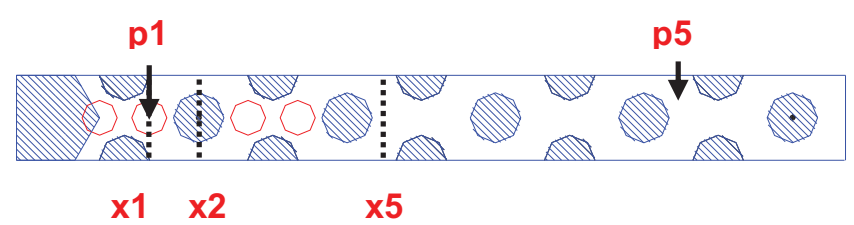

Figure 3. Locations of points and profiles.

An indication of the large amplitude fluctuations associated with the random instability of the four-jet case can be seen in time signatures at points. Figure 4 plots the instantaneous time signature of the vertical velocity $w$ at point $\mathrm{p} 1 \mathrm{u}$ for the MIR data for the four-jet case. As can be seen, the amplitude of the fluctuations is quite large, ranging from about -9 to $1 \mathrm{~m} / \mathrm{sec}$. For comparison, Figure 5 plots the time trace of the ensembleaveraged velocity $\langle w\rangle$ at plu for the AKN and SKW turbulence models also for the four-jet case. The range of fluctuation is seen to be from about -9 to -2.5 , a somewhat lower magnitude than for the MIR data, but still with a very large variation in amplitude.

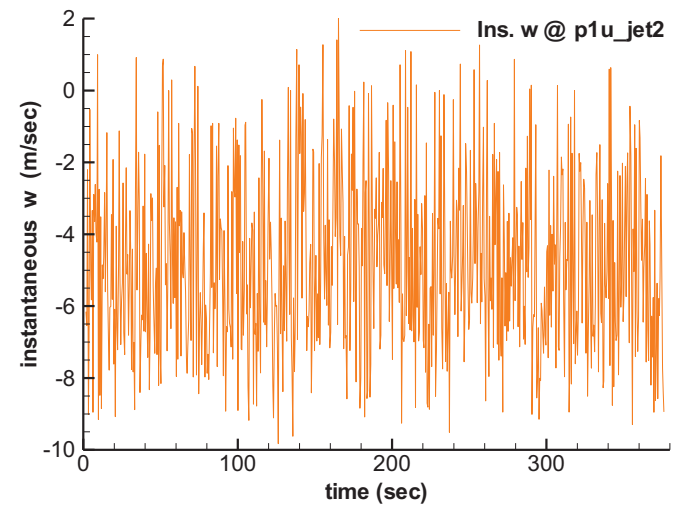

Figure 4. Time trace of $w$ at $p 1 \mathrm{u}$ for MIR four-jet data.

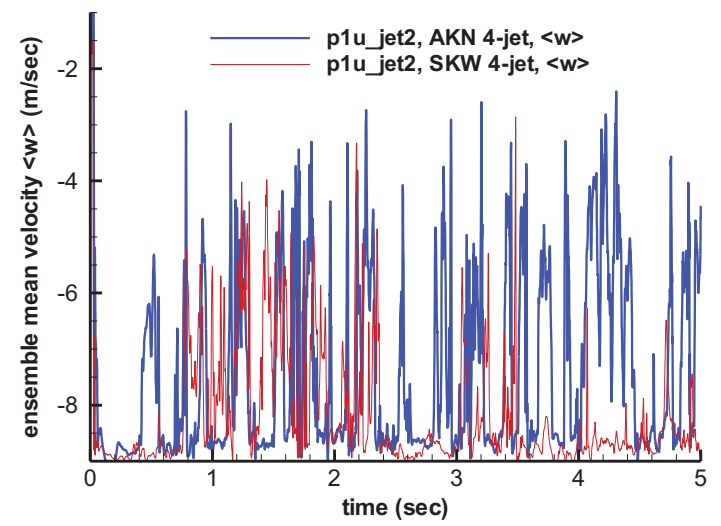

Figure 5. Trace of $\langle w\rangle$ at $\mathrm{p} 1 \mathrm{u}$ for 4 -jet computations. 
However, when Jet 1 is shut off, the flow behavior changes dramatically. Figure 6 plots the results for the four turbulence models for the three-jet case for $\langle w\rangle$ at point p1u, the same point as for Figs. $4 \& 5$ above. As can be seen, the amplitude of the fluctuation for the ensemble-averaged velocity $\langle w\rangle$ has decreased to very small ranges after the initial transient, which started from static conditions. It is this change in the flow behavior that is hoped represents a stabilization of the unstable recirculation zone below Jets 1 and 2 from the four-jet case.

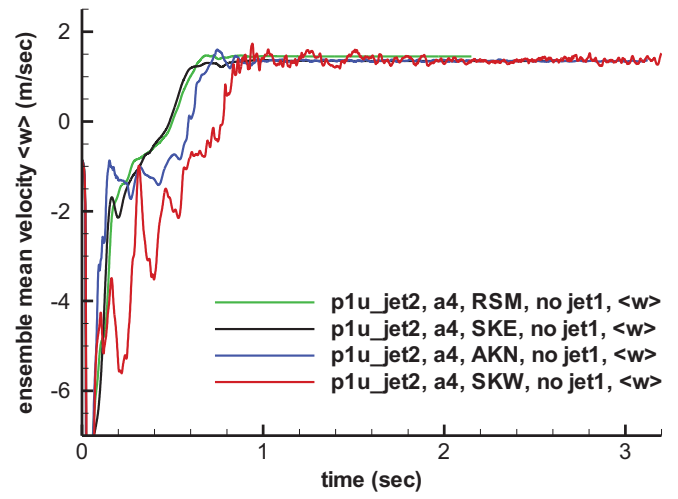

Figure 6. Trace of $\langle w\rangle$ at $p 1 u$ for 3 -jet computations.

Figure 7 plots the streamlines for the original four-jet case and for the new three-jet case for the SKW model. As can be seen for the four-jet case, the recirculation zone below Jet 1 flows back and impinges on itself with some energy, causing the separation point to move violently up and down as plots at other times show [4]. For the three-jet case, the recirculation zone occupies the whole height of the model and remains stable as plots at other times show [4].
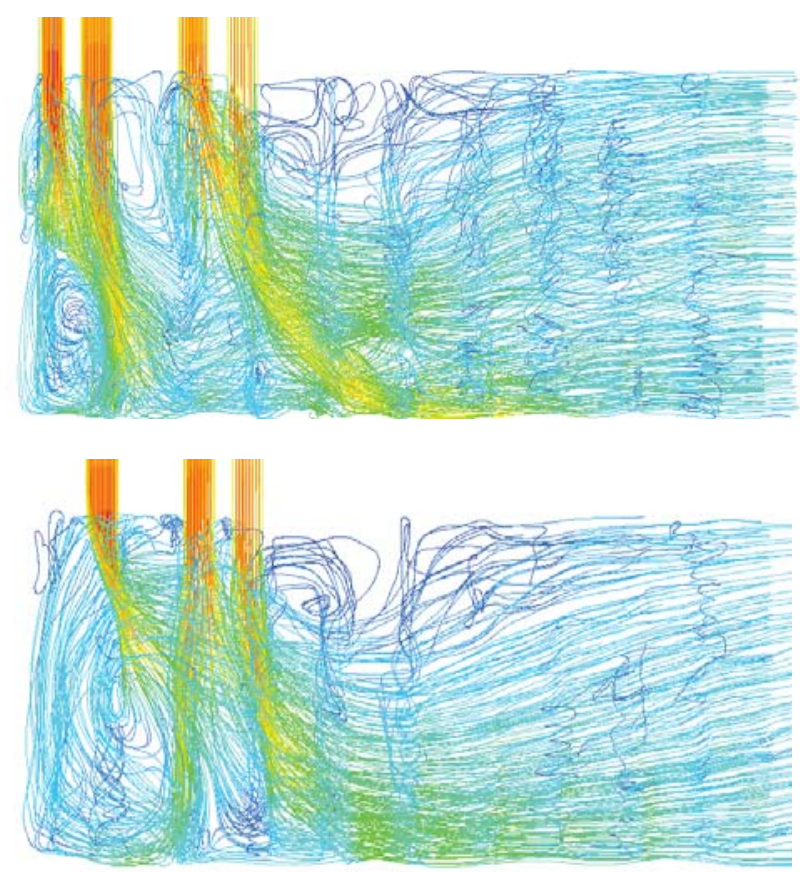

Figure 7. Streamline plot for SKW model for four-jet (upper) case and three-jet case.
Figure 8 plots the horizontal ensemble-averaged velocity $\langle u\rangle$ for the three-jet case for the four turbulence models at point $\mathrm{p} 5 \mathrm{~d}$. This point is rather far from the inlet jets where the flow is nearly horizontal (see Figs. 3, 7) and should be showing vortex shedding. The time traces for the RSM and SKE models, however, show the velocity reaching a steady state after the initial transient without vortex shedding. The trace for the AKN model show some regular fluctuations, but with low amplitude. It is not clear what kind of behavior this represents. Because the amplitude is so low, it could be related to pressure fluctuations but without actual vortex-shedding. Finally, the results for the SKW model indicate vortex-shedding with fairly large amplitude variations. It is shown in Ref. [6] that the four turbulence models used show increasingly greater levels of turbulent viscosity from the SKW to the AKN to the SKE to the RSM models for the same flow. This helps explain the range of fluctuations in the results in Fig. 8.

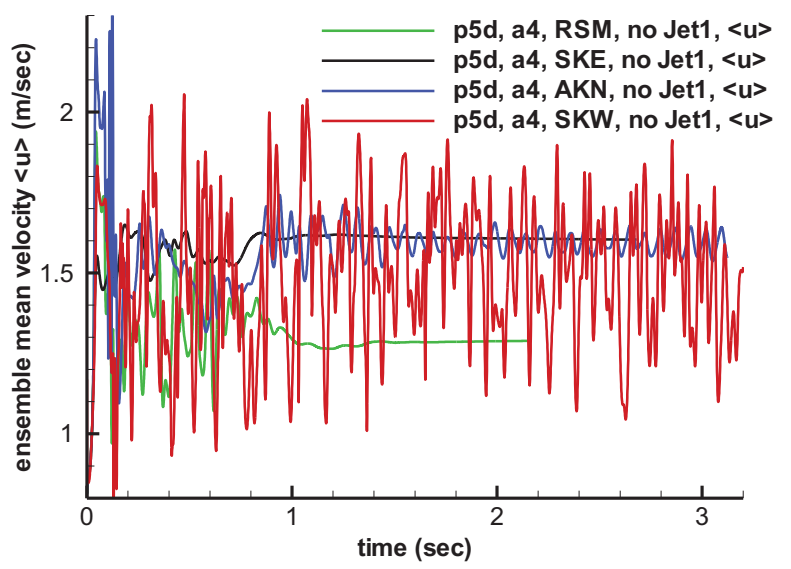

Figure 8. Trace of $\langle u\rangle$ at $\mathrm{p} 5 \mathrm{~d}$ for 3 -jet computations.

Long-time averaged results for several velocity profiles are compared for the three-jet case against the four-jet MIR data. These represent blind predictions. The long-time averages were initiated after the initial transients shown in Fig. 6. Figure 9 illustrates time mean vertical velocity $W$ for locations $\mathrm{x} l \mathrm{u}$ and x1d. The predictions indicate positive $W$ or upflow, in accord with the large recirculation zone shown in Fig. 7. Results for the four turbulence models are close except for the SKW model, which is somewhat lower.

Figure 10 shows time mean horizontal velocity $U$ also at $\mathrm{x} 1 \mathrm{u}$ and $\mathrm{x} 1 \mathrm{~d}$. The velocity is positive at $\mathrm{x} 1 \mathrm{u}$ and negative at $\mathrm{x} 1 \mathrm{~d}$, which also accords with the recirculation zone. At $\mathrm{x} 1 \mathrm{u}$, the SKE model shows a slightly different trend than the others with a maximum in the center. The trends are the same for $\mathrm{xld}$, though the SKW results are somewhat lower.

Figure 11 plots results for $U$ at $\mathrm{x} 2 \mathrm{u}$ and $\mathrm{x} 2 \mathrm{~d}$. The three-jet results are the reverse of the four-jet data. The velocity is positive at $\mathrm{x} 2 \mathrm{u}$ and negative at $\mathrm{x} 2 \mathrm{~d}$, which again matches the recirculation zone in Fig. 7. Figure 12 illustrates results for $U$ at $\mathrm{x} 5 \mathrm{u}$ and $\mathrm{x} 5 \mathrm{~d}$. At $\mathrm{x} 5 \mathrm{u}, U$ is seen to be predicted to be either small or negative for all but the SKW model. At $\mathrm{x} 5 \mathrm{~d}$, the flow is seen to be strongly horizontal for all the predictions and for the data. 

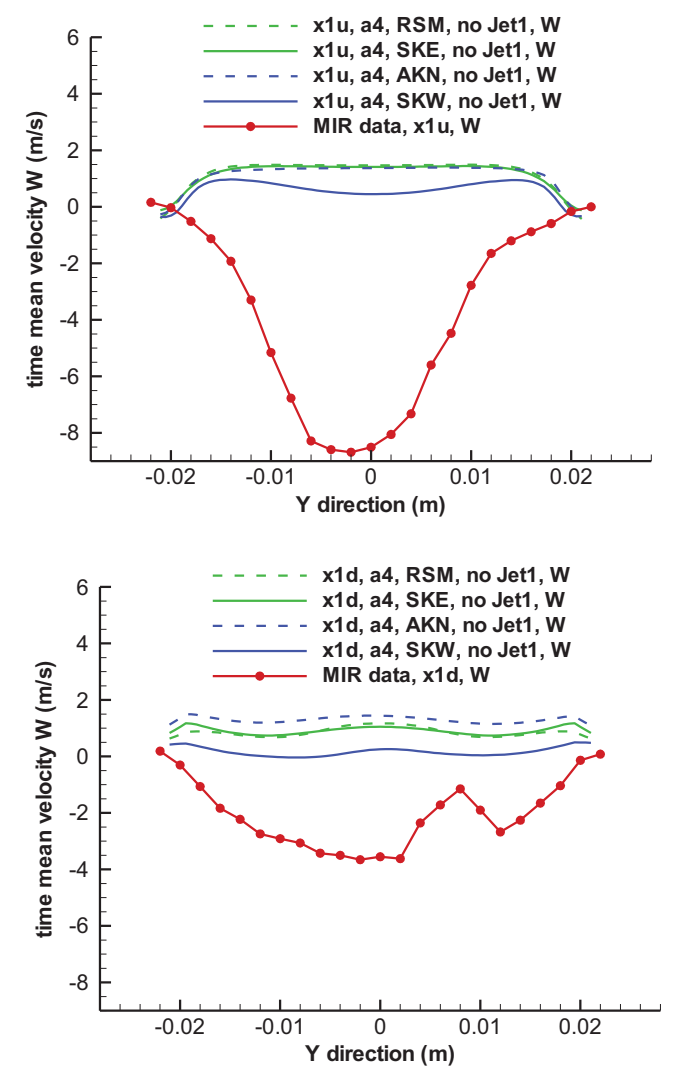

Figure 9. Time mean velocity $W$ at $\times 1 \mathrm{u}$ and $\times 1 \mathrm{~d}$.
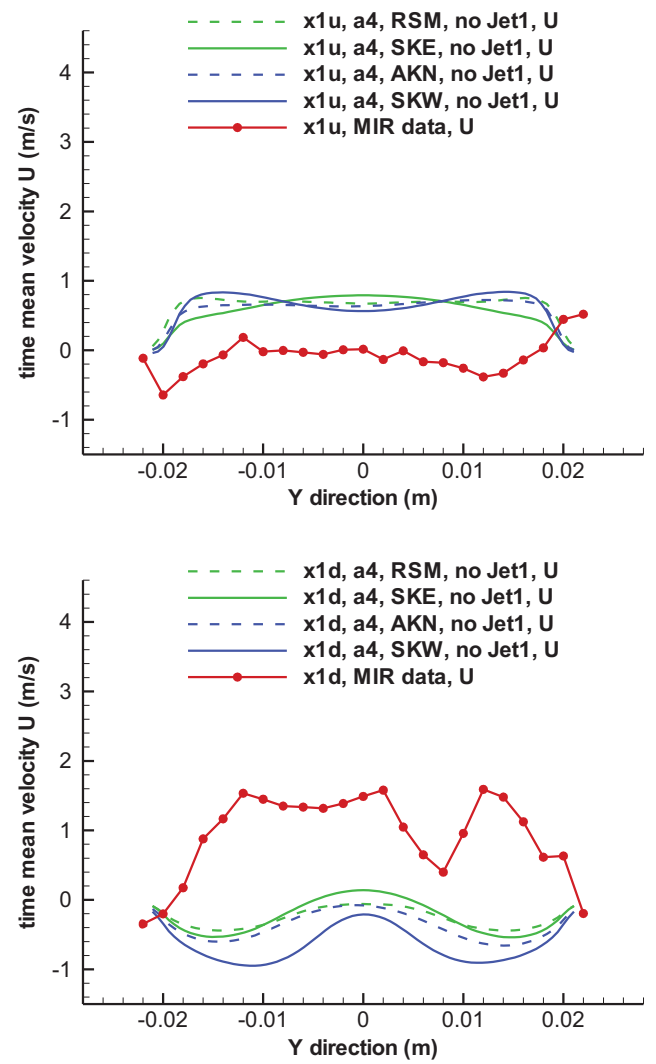

Figure 10. Time mean velocity $U$ at $x 1 \mathrm{u}$ and $\mathrm{x} 1 \mathrm{~d}$.
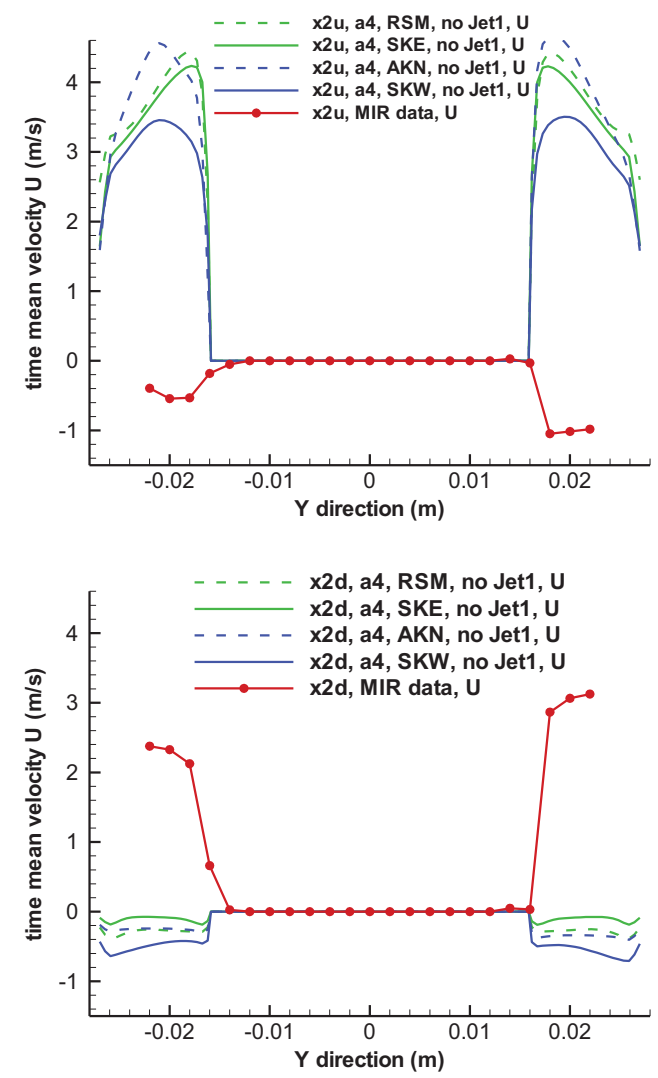

Figure 11. Time mean velocity $U$ at $\times 2 u$ and $\times 2 d$.
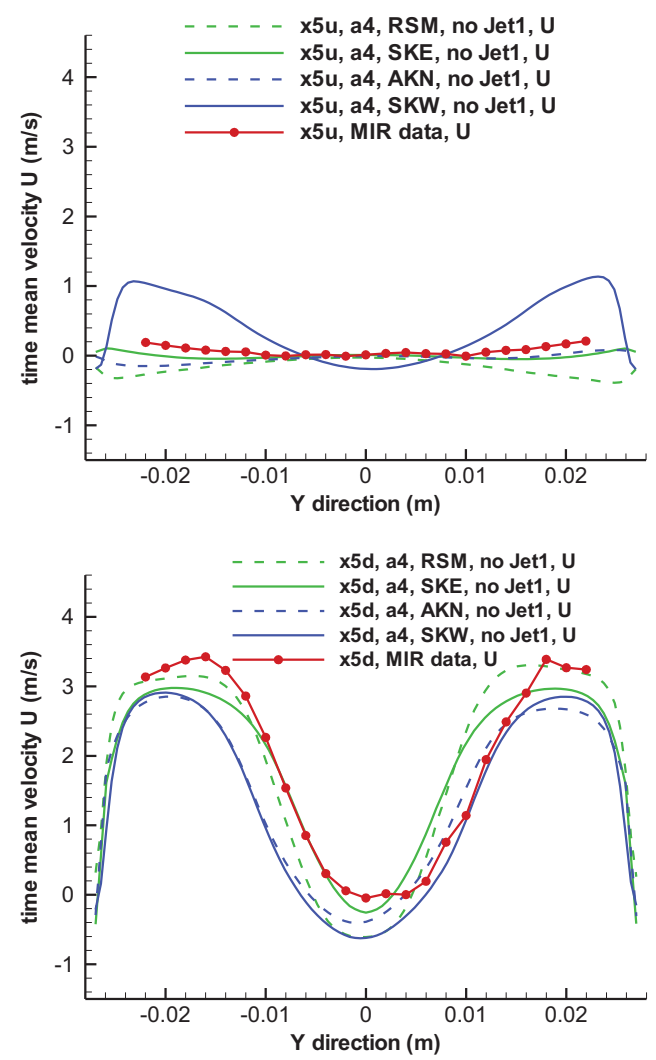

Figure 12. Time mean velocity $W$ at $\times 1 \mathrm{u}$ and $\mathrm{x} 1 \mathrm{~d}$. 
Additional views of the flow field are given by contour plots of the vertical velocity for two vertical planes, $Z=-70$ (upper) and $-150 \mathrm{~mm}$ (lower). Figure 13 shows the time mean velocity $W$ at these two planes for the SKW turbulence model. The other models show similar results. Note that the scale is different for the two plots. For the upper plane, the signatures of the three jets are obvious and symmetric. The leftmost jet is wrapped around the first full cylinder; the next jet is squeezed laterally by the converging flow and the last jet is expanded in the lateral direction by the diverging flow. At the lower plane, the signature of the leftmost jet has disappeared; the second jet is greatly diminished and the last jet is now wrapped around the second full cylinder.
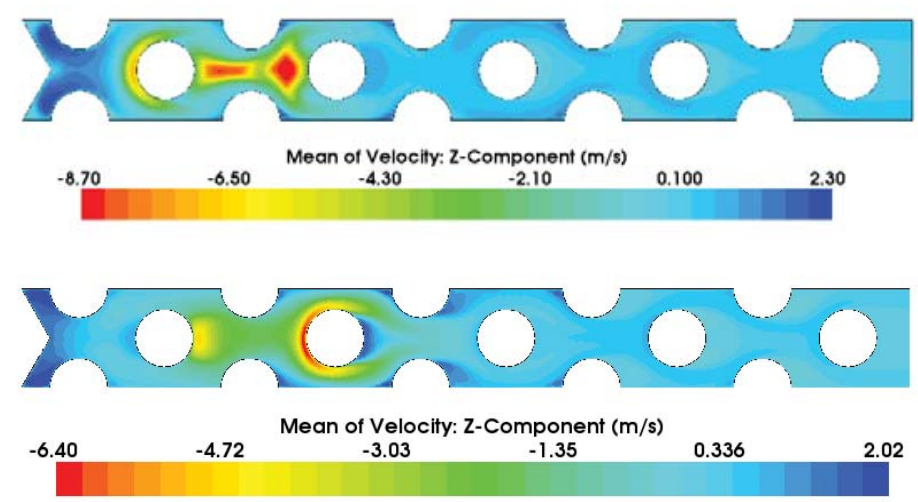

Figure 13. Contours of $W$ for $Z=-70$ and $-150 \mathrm{~mm}$.

\section{SUMMARY}

Blind predictions have been made for a reconfigured experiment based on an earlier test that employed a scaled model of a slice of the lower plenum of a very high temperature reactor (VHTR). The predicted flow is isothermal with only three inlet jets, instead of four. It was determined that the flow field for the four inlet jet configuration included a very unstable recirculation zone that has been determined to be undesirable for a validation data set. Elimination of the first jet appears to have stabilized and increased the size of the unstable recirculation zone, according to computations. It has been recommended that new data be taken with the first jet shut off; these data are yet forthcoming. The flow also includes a number of half and full cylinders that represent support columns in the lower plenum that hold up the core. It is believed that there is vortex shedding around the support pillars, making the turbulent flow quite complex. Along with obtaining inlet data with improved accuracy using particle image velocimetry, it is anticipated that the new three-jet data will be suitable for validation data for computational fluid dynamics for application to nuclear reactor safety analysis in the VHTR.

\section{NOMENCLATURE}

AKN Abe-Kondoh-Nagano low Re $k \sim \varepsilon$ turbulence model

INL Idaho National Laboratory, Idaho Falls, ID

$k \quad$ turbulent kinetic energy

$\begin{array}{ll}\text { MIR } & \text { matched index of refraction facility } \\ \text { NGNP } & \text { next generation nuclear plant } \\ \text { PIV } & \text { particle image velocimetry } \\ \text { SKE } & \text { standard } k \sim \varepsilon \text { turbulence model } \\ \text { SKW } & \text { Menter (SST) } k \sim \omega \text { turbulence model } \\ \text { RSM } & \text { Reynolds stress transport turbulence model } \\ \text { TKE } & \text { turbulent kinetic energy } \\ \text { URANS } & \text { unsteady Reynolds-averaged Navier-Stokes } \\ \text { VHTR } & \text { very high temperature reactor } \\ y^{+} & \text {dimensionless wall distance } \\ \varepsilon & \text { turbulent energy dissipation rate } \\ \omega & \text { specific turbulent dissipation rate }\end{array}$

\section{ACKNOWLEDGMENTS}

Work supported by the U.S. Department of Energy, Office of Nuclear Energy, under DOE Idaho Operations Office Contract DE-AC07-05ID14517.

\section{REFERENCES}

[1] McIlroy, H. M., D. M. McEligot and R. J. Pink, "Measurement of Flow Phenomena in a Lower Plenum Model of a Prismatic Gas-Cooled Reactor," Proceedings of the $16^{\text {th }}$ International Conference on Nuclear Engineering (ICONE-16), Orlando, May 11-15, 2008.

[2] STAR-CCM+, version 3.04, 2008, CD-adapco, 60 Broadhollow Road, Melville, NY 11747.

[3] Johnson, R. W., "Examination of a Proposed Validation Data Set Using CFD Calculations," Proceedings of ASME 2009 Fluids Engineering Division Summer Meeting, paper FEDSM2009-78317, Vail, Colorado, August 2-5, 2009.

[4] Johnson, R. W., H. M. McIlroy, R. C. Johnson and D. P. Christensen, Undesirable Flow Behavior in a Proposed Validation Data Set, Paper ICONE18-29474, Proceedings of the 18th International Conference on Nuclear Engineering (ICONE18), May 17-21, 2010, Xi'an, China.

[5] R. W. Johnson, Development of a CFD Analysis Plan for the First VHTR Standard Problem, Proceedings of the $4^{\text {th }}$ International Topical Meeting on High Temperature Reactor Technology (HTR2008), paper HTR2008-5828, Washington, D. C., Sept. 28 - Oct. 1 (2008).

[6] Johnson, R. W., "Evaluation of an Experimental Data Set to be Validation Data for CFD for a VHTR," CFD4NRS-3 conference, Sept. 14-16, 2010, Washington, D.C.

[7] FLUENT, version 6.3.26, 2008. FLUENT Inc., 10 Cavendish Court, Centerra Resource Park, Lebanon, NH, 03766.

[8] Johnson, R. W. and Schultz, R. R., "Bounding Estimate for the 'Hot' Channel Coolant Temperature and Preliminary Calculation of Mixing in the Lower Plenum for the NGNP Point Design Using CFD," Report INEEL/EXT-04-02570, Rev. 1, Idaho National Engineering \& Environmental Laboratory, December 2004.

[9] Johnson, R. W., "Modeling Strategies for Unsteady Turbulent Flows in the Lower Plenum of the VHTR," Nuclear Engineering \& Design, 238, 482-491, 2008. 